\title{
Standardbezogene Kompetenzen im Fach Mathematik am Ende der gymnasialen Oberstufe
}

Ein Vergleich über eine Linking-Studie

\author{
Nele Kampa $\mathbb{D} \cdot$ Hannah Hinz $\cdot$ Nicole Haag $\cdot$ Olaf Köller
}

Online publiziert: 5. September 2017

(C) Der/die Autor(en) 2017. Dieser Artikel ist eine Open-Access-Publikation.

Zusammenfassung Mittlerweile liegen verschiedene Schulleistungsstudien vor, in denen die Mathematikkompetenzen am Ende der gymnasialen Oberstufe bestimmt wurden. Generell zeigt sich, dass die Ziele des voruniversitären Mathematikunterrichts von mehr als der Hälfte der Abiturientinnen und Abiturienten verfehlt werden. Basierend auf diesen Befunden gehen wir der Frage nach, ob zumindest die mit den Bildungsstandards für den Mittleren Schulabschluss (MSA) verbundenen Ziele im Fach Mathematik am Ende der gymnasialen Oberstufe erreicht werden. Trägt also die gymnasiale Oberstufe dazu bei, dass die Inhalte und Kompetenzen der Sekundarstufe I im voruniversitären Unterricht konsolidiert, besser noch, vertieft werden? Dazu wurden die Daten von $N=3641$ Schülerinnen und Schülern ausgewertet, die eine 13. Jahrgangsstufe eines allgemeinbildenden oder beruflichen Gymnasiums in Schleswig-Holstein besucht hatten. Der verwendete Mathematiktest wurde in einem Skalenlinking auf die nationale Metrik $(M=500, S D=100$ am Ende der

Zusatzmaterial online Zusätzliche Informationen sind in der Online-Version dieses Artikels (https:// doi.org/10.1007/s11618-017-0777-8) enthalten.

Dr. N. Kampa $(\bowtie) \cdot$ Prof. O. Köller

Leibniz-Institut für die Pädagogik der Naturwissenschaften und Mathematik (IPN),

Olshausenstraße 62, 24118 Kiel, Deutschland

E-Mail: kampa@ipn.uni-kiel.de

Prof. O. Köller

E-Mail: koeller@ipn.uni-kiel.de

H. Hinz

Abteilung: Sozialpädagogik, Berufsbildungszentrum Schleswig, Flensburger Straße 19b, 24837 Schleswig, Deutschland

E-Mail: hannah.hinz@freenet.de

Dr. N. Haag

Institut zur Qualitätsentwicklung im Bildungswesen (IQB), Unter den Linden 6, 10099 Berlin,

Deutschland

E-Mail: nicole.haag@iqb.hu-berlin.de 
9. Jahrgangsstufe) des IQB-Ländervergleichs 2012 für den MSA transformiert. Die Ergebnisse zeigen für verschiedene Profile an allgemeinbildenden Gymnasien und Zweige an beruflichen Gymnasien, dass die Erwartungen, die typischerweise an den Erwerb des MSA gestellt werden, deutlich überschritten werden. Implikationen für die Zielsetzungen voruniversitärer Bildung werden abgeleitet.

Schlüsselwörter Bildungsstandards · Large-scale Assessment · Gymnasiale Oberstufe · Mathematikkompetenzen · Profiloberstufe

\title{
Standard-based abilities in mathematics at the end of upper secondary education
}

A linking-study

\begin{abstract}
Several large-scale assessments focus on mathematical abilities at the end of upper secondary education. They show that more than $50 \%$ of graduates fail to meet the target-level of pre-university education in mathematics. To extend these findings, we pose the question whether the same graduates at least meet the goals related to the Educational Standards for the intermediate school-leaving certificate. Particularly, we investigate whether the upper secondary education succeeds in consolidating or even deepening the content and abilities of the lower secondary level education. We analyzed data from students $(N=3641)$ who were enrolled in grade 13 in academic and vocational upper secondary schools in Schleswig-Holstein. In upper secondary level in Schleswig-Holstein, students opt between different tracks: academic and vocational. Through a linking study, the administered test in mathematics was transformed to the national metric $(M=500, S D=100$, end of grade 9) of the IQB-National Assessment 2012 for the intermediate school leaving certificate in mathematics. The results show that at the end of upper secondary education norms that are typically expected for the acquisition of the intermediate school leaving certificate are more than met in almost all tracks. We conclude by elucidating implications for target levels of pre-university education.
\end{abstract}

Keywords Educational Standards · Large-scale assessment - Mathematical ability · Upper secondary education

\section{Einleitung}

Seit dem Erscheinen der Befunde der Dritten Internationalen Mathematik- und Naturwissenschaftsstudie (TIMSS; Baumert et al. 2000a, 2000b) sind verschiedene weiterführende Untersuchungen durchgeführt worden, in denen die Mathematikkompetenzen deutscher Schülerinnen und Schüler am Ende der gymnasialen Oberstufe festgestellt wurden (Köller et al. 2004; Trautwein et al. 2007, 2010; Leucht et al. 2016). Den Studien ist gemeinsam, dass deutlich über $50 \%$ der Schülerinnen und Schüler viele Aufgaben, die Inhalte der gymnasialen Oberstufe thematisieren, nicht mit hinreichender Sicherheit lösen können. In der kürzlich publizierten Arbeit von Kampa et al. 2016a wiesen in Schleswig-Holstein $70 \%$ der Schülerschaft an 
allgemeinbildenden Gymnasien unmittelbar vor der Abiturprüfung unzureichende Mathematikkenntnisse auf.

Da die Befunde nahelegen, dass möglicherweise auch die Inhalte der Sekundarstufe I nicht sicher beherrscht werden, gehen wir der Frage nach, welche Kompetenzen Abiturientinnen und Abiturienten an allgemeinbildenden und beruflichen Gymnasien in Schleswig-Holstein mit Bezug zu den Bildungsstandards für den Mittleren Schulabschluss (MSA; vgl. Pant et al. 2013) erreichen. Dazu führen wir Sekundäranalysen der Studie Lernergebnisse an allgemeinbildenden und beruflichen Gymnasien in Schleswig-Holstein (LISA 6; Leucht et al. 2016) durch. In LISA 6 wurden Mathematikkompetenzen mit einem Test aus dem Nationalen Bildungspanel (NEPS) erhoben. Dieser Test wurde in einer Zusatzstudie mit normierten Items aus dem Ländervergleich 2012 des Instituts zur Qualitätsentwicklung im Bildungswesen (IQB-LV 2012; vgl. Pant et al. 2013) verlinkt. Damit können die Leistungen der Abiturientinnen und Abiturienten aus LISA 6 mit der nationalen Skala zur Überprüfung der Erreichung der MSA-Bildungsstandards im Fach Mathematik verbunden werden. Zunächst beschreiben wir das im IQB-LV 2012 verwendete Kompetenzstrukturmodell und Kompetenzstufenmodell sowie das Abschneiden der Schülerinnen und Schüler Schleswig-Holsteins im IQB-LV 2012 (Pant et al. 2013) und arbeiten bisherige Befunde zu den Mathematikkompetenzen am Ende der gymnasialen Oberstufe auf. Die Ergebnisse münden dann in eine Diskussion, in der wir die Sicherung grundlegender mathematischer Kompetenzen als Kernziel der gymnasialen Oberstufe beschreiben.

\section{Kompetenzstruktur- und Kompetenzstufenmodell der Bildungsstandards für den MSA im Fach Mathematik}

Im Rahmen der Entwicklung der Bildungsstandards für den MSA wurde ein Kompetenzstrukturmodell vorgeschlagen (siehe Abb. 1), welches eine Prozess-, eine Inhalts- und eine Anspruchsdimension spezifiziert (Roppelt et al. 2013).

Die sechs prozessbezogenen Kompetenzen sind: (1) Argumentieren, (2) Probleme lösen, (3) Modellieren, (4) Darstellungen verwenden, (5) mit symbolischen, formalen und technischen Elementen umgehen sowie (6) Kommunizieren (KMK 2003; Blum 2006). Auf der inhaltlichen Dimension befinden sich die fünf Leitideen Zahl, Messen, Raum und Form, funktionaler Zusammenhang sowie Daten und Zufall. Des Weiteren werden die drei Anforderungsbereiche (1) Reproduzieren, (2) Zusammenhänge herstellen und (3) Verallgemeinern und Reflektieren unterschieden.

Zur schrittweisen Einteilung der Kompetenzausprägungen werden konkrete normative und abschlussbezogene Erwartungen an Schülerinnen und Schüler formuliert. Kompetenzstufenmodelle befassen sich in diesem Zusammenhang mit der inhaltlichen Beschreibung von empirisch erhobenen Kompetenzwerten, die eine kriteriumsorientierte Interpretation dieser Testwerte ermöglicht (Goldhammer und Hartig 2007). Hierfür wird durch Standard-Setting-Verfahren eine kontinuierliche Skala in inhaltlich sinnvolle Abschnitte unterteilt - die Kompetenzstufen - welche mit den jeweiligen Anforderungen in diesem Leistungsbereich verknüpft sind (Hartig und Klieme 2006; Klieme et al. 2007; Gniewosz 2015). Zu diesem Zweck liegt für die 
Abb. 1 Die drei Dimensionen mathematischer Kompetenzen in den Bildungsstandards. (Aus: IQB 2012)

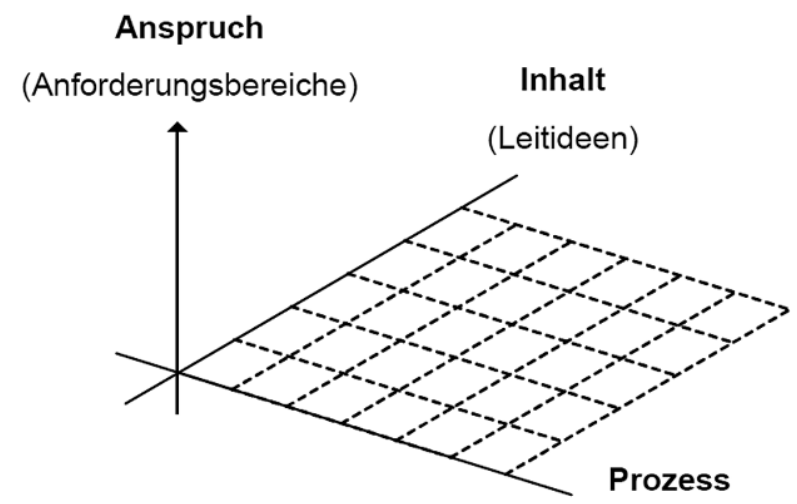

(Kompetenzen)

MSA-Bildungsstandards in Mathematik ein Kompetenzstufenmodell vor, das sowohl für die globale Mathematikkompetenz als auch für jede Leitidee beschrieben wird. Tabelle 1 zeigt die Stufenbereiche für das Globalmodell (Kompetenzstufenbeschreibungen siehe online verfügbares Zusatzmaterial).

Der Mindeststandard legt ein Minimum an Kompetenzen fest, welches alle Schülerinnen und Schüler, die einen MSA anstreben, bis zum Ende der Sekundarstufe I erreicht haben sollten. Der darüber liegende Regelstandard beschreibt einen Leistungsbereich, der Erwartungen abbildet, die an Schülerinnen und Schüler gestellt werden, wenn diese den MSA erwerben. Ferner wurde mit dem Regelstandard plus ein Leistungsbereich definiert, der über diese Erwartungen hinausgeht, strenggenommen aber sogar das eigentliche Ziel des Unterrichts sein sollte. Der Optimalstandard beschreibt Kompetenzen, die unter sehr guten individuellen Lernvoraussetzungen und Lerngelegenheiten erzielt werden können und deutlich über den Erwartungen der Bildungsstandards liegen (IQB 2012).

\section{3 (Standardbezogene) Mathematikkompetenzen am Ende der Sekundarstufen I und II}

Basierend auf den MSA-Bildungsstandards im Fach Mathematik hat das IQB 2013 den ersten Ländervergleich publiziert. Die Ergebnisse erlauben eine Verortung von Neuntklässlerinnen und -klässlern auf einer nationalen Mathematikskala $(M=500$ und $S D=100$ ). Schülerinnen und Schüler der 9. Jahrgangsstufe in Schleswig-Holstein lagen im Jahr 2012 bei $M=502$ Punkten, die Gymnasiastinnen und Gymnasiasten bei $M=587$ Punkten (nationaler Mittelwert $M=586$ ). Bezogen auf die Kompetenzstufen ergab sich die in Tab. 2 dargestellte Verteilung.

Die Erwartungen an den MSA wurden demnach bundesweit von 56\% der Schülerinnen und Schüler am Ende der 9. Jahrgangsstufe verfehlt (in Schleswig-Holstein $54 \%$ ). Am Gymnasium lagen die entsprechenden Zahlen bei 17 bzw. $14 \%$.

Für die Sekundarstufe II liegen mittlerweile einige Befunde zu Kompetenzen im Fach Mathematik vor. In der TIMSS-Studie (Baumert et al. 2000b) wurden Schü- 
Tab. 1 Mindest-, Regel- und Maximalstandards in Mathematik für den Mittleren Schulabschluss (MSA)

\begin{tabular}{lll}
\hline Kompetenzstufen & Schwellenwerte & MSA Standard \\
\hline V & 675 und darüber & Optimalstandard \\
IV & 595 bis 674 & Regelstandard plus \\
III & 515 bis 594 & Regelstandard \\
II & 435 bis 514 & Mindeststandard \\
I.b & 355 bis 434 & Unter Mindeststandard \\
I.a & Unter 355 & \\
\hline
\end{tabular}

Tab. 2 Verteilungen der Neuntklässlerinnen und -klässler auf die Kompetenzstufen in Mathematik nach Bildungsgang und regionaler Herkunft (Angaben in Prozent)

\begin{tabular}{lllll}
\hline & Bundesweit & & \multicolumn{2}{l}{ Schleswig-Holstein } \\
& Alle SuS & SuS am Gymnasium & Alle SuS & SuS am Gymnasium \\
\hline Stufe I & 25,0 & 1,8 & 23,4 & 1,0 \\
Stufe II & 30,7 & 14,9 & 30,3 & 12,8 \\
Stufe III & 25,9 & 39,0 & 28,3 & 42,2 \\
Stufe IV & 14,2 & 33,1 & 14,3 & 34,1 \\
Stufe V & 4,2 & 11,3 & 3,7 & 9,9 \\
\hline
\end{tabular}

SuS Schülerinnen und Schüler

lerinnen und Schüler der gymnasialen Oberstufe auf Kompetenzstufen verortet. Die Kompetenzstufe I spiegelt Anforderungen am Ende der Primarstufe, die Kompetenzstufe II Anforderungen der Sekundarstufe I und die Kompetenzstufen III und IV voruniversitäre mathematische Kompetenzen wider. Nur $30 \%$ der Schülerinnen und Schüler in TIMSS lagen mindestens auf Kompetenzstufe III; somit erreichten $70 \%$ nicht die Lernziele der voruniversitären Mathematik.

Abiturientinnen und Abiturienten an allgemeinbildenden und beruflichen Gymnasien in Baden-Württemberg waren der Untersuchungsgegenstand des Projekts Transformation des Sekundarschulsystems und akademische Karrieren (TOSCA, Köller et al. 2004). Die untersuchten Schülerinnen und Schüler wurden nach einem Kurssystem beschult und bearbeiteten den TIMSS-Test zu voruniversitärerer Mathematik. An allgemeinbildenden Gymnasien erreichte ca. die Hälfte der Schülerschaft nicht die Ziele voruniversitärer mathematischer Bildung (Kompetenzstufen III und IV). Bei einem Vergleich von allgemeinbildenden und beruflichen Gymnasien zeigten sich starke Differenzen zwischen den beiden Schulformen, welche einem Leistungsvorsprung von ca. eineinhalb Schuljahren entsprachen (Watermann et al. 2004). Die Ergebnisse auf Ebene der Zweige an beruflichen Gymnasien zeigten, dass lediglich Schülerinnen und Schüler im technischen Zweig vergleichbare Kompetenzen wie an allgemeinbildenden Gymnasien erreichten. Die großen Unterschiede konnten teilweise durch unterschiedliche kognitive Grundfähigkeiten der Schülerinnen und Schüler erklärt werden.

Nach einer Reform, die zu einer Profiloberstufe führte, fand die Folgeuntersuchung TOSCA-Repeat statt. Erneut wurden die voruniversitären Kompetenzen von Abiturientinnen und Abiturienten an allgemeinbildenden Schulen mit dem gleichen TIMSS-Test untersucht. Im Profilsystem wurden alle Schülerinnen und Schüler auf 
erhöhtem Anforderungsniveau in Mathematik unterrichtet (vier Stunden). Der geringe Mittelwertunterschied im Vergleich zur Vorgängerstudie legt nahe, dass auch hier fast $50 \%$ der Schülerinnen und Schüler Standards der voruniversitären Mathematik nicht erreichen (unter Kompetenzstufe III).

In der Studie Aspekte der Lernausgangslagen und Lernentwicklung (LAU) wurde für einen Vergleich mit Baden-Württemberg erneut der TIMSS-Test für voruniversitäre Mathematik im gesamten Abiturjahrgang 2004/05 in Hamburg eingesetzt, um dessen Kompetenzen mit denen in Baden-Württemberg zu vergleichen (Trautwein et al. 2007). Als Zielerwartung für Grundkurse wurde die Kompetenzstufe II, für Leistungskurse die Kompetenzstufen III und IV definiert. In Hamburg erreichten $55 \%$ der Schülerinnen und Schüler in Leistungskursen diesen Standard gegenüber $83 \%$ in Baden-Württemberg. Für die Grundkurse lagen die entsprechenden Anteile bei $52 \%$ in Hamburg und $86 \%$ in Baden-Württemberg.

In der LISA 6-Studie wurden mit dem Grundbildungstest, der in NEPS zu Beginn des Studiums eingesetzt wird, erneut mathematische Kompetenzen von Schülerinnen und Schülern am Ende der gymnasialen Oberstufe in einem Profilsystem untersucht. In der Profiloberstufe belegen die Schülerinnen und Schüler Mathematik, Deutsch, eine Fremdsprache und ein profilgebendes Fach auf erhöhtem Anforderungsniveau (vierstündig). Fünf Profile (naturwissenschaftlich, sprachlich, gesellschaftswissenschaftlich, sportlich, ästhetisch) legen darüber hinaus Schwerpunkte fest (bspw. Belegung von insgesamt 10 Wochenstunden in den Fächern Biologie, Physik und Chemie im naturwissenschaftlichen Profil).

An beruflichen Gymnasien existieren analog die Zweige Technik, Wirtschaft, Ernährung, Agrarwissenschaft, Pädagogik und Psychologie sowie Gesundheit, die auch mit fachlichen Schwerpunktsetzungen verbunden sind. In LISA 6 zeigte sich, dass die mathematischen Kompetenzen in der 13. Jahrgangsstufe je nach Schulform sowie Profil bzw. Zweig und Unterrichtszeit stark variierten. Schülerinnen und Schüler, die vierstündig auf einem allgemeinbildenden Gymnasium unterrichtet werden, erreichten die höchsten Kompetenzen, gefolgt von Schülerinnen und Schülern an beruflichen Gymnasien mit fünfstündiger Unterrichtszeit. Die geringsten mathematischen Kompetenzen zeigten Schülerinnen und Schüler, die an beruflichen Gymnasien mit nur drei Stunden unterrichtet werden (Kampa et al. 2016a). Wird die mathematische Kompetenz für die kognitiven Grundfähigkeiten kontrolliert, reduzieren sich die Unterschiede zwischen den drei Gruppen, sie verschwinden jedoch nicht. Weitere Unterschiede zeigten sich in LISA 6 auch bezüglich der Profile und Zweige. Die mit großem Abstand höchsten Kompetenzen sind im naturwissenschaftlichen Profil zu finden. Die Schülerinnen und Schüler im Zweig Technik zeigen ähnlich hohe mathematische Kompetenzen wie ihre Mitschülerinnen und Mitschüler im sprachlichen, gesellschaftswissenschaftlichen und sportlichen Profil an allgemeinbildenden Gymnasien. Die geringsten Kompetenzen zeigen Schülerinnen und Schüler im Profil Ernährung. Diese Unterschiede in den Kompetenzen spiegeln sich auch in dem Erreichen von Standards wieder.

In der LISA 6-Studie wurde auf Grundlage des NEPS-Tests ein Standard-Setting durchgeführt (Kampa et al. in Vorbereitung). Das resultierende Kompetenzstufenmodell wurde auf theoretischer Ebene mit den bereits bestehenden Kompetenzstufenmodellen für die Sekundarstufe II verbunden (Kampa et al. 2016b), so dass auch 
auf dem neu entwickelten Modell das Erreichen von Standards in der Oberstufe auf der Kompetenzstufe III angesiedelt wurde. Während am allgemeinbildenden Gymnasium und an beruflichen Gymnasien bei fünfstündigem Mathematikunterricht 31 bzw. $26 \%$ die Kompetenzstufe III erreichen, sind es bei dreistündiger Unterrichtung am beruflichen Gymnasium nur knapp über $5 \%$.

\section{Zuwächse in der gymnasialen Oberstufe}

In der LAU-Studie wurden die Kompetenzen von Hamburger Schülerinnen und Schülern im Längsschnitt getestet (Behörde für Schule und Berufsbildung 2012). Zum Einsatz kamen Aufgaben zur mathematischen Grundbildung, die nicht explizit Inhalte der gymnasialen Oberstufe thematisierten. Die Ergebnisse in Tab. 3 machen sichtbar, dass die Zuwächse innerhalb von drei Schuljahren insgesamt etwas über einem Drittel einer $S D$ liegen. In Grundkursen fallen die Zuwächse etwas geringer aus, in Leistungskursen erreichen sie zwei Drittel bis eine $S D$.

Etwas günstigere Befunde zeigten Analysen zur Veränderung der Mathematikkompetenzen vom Ende der 10. bis zum Ende der 12. Jahrgangsstufe auf der Basis der BIJU-Studie (Köller et al. 2010). Diesen Analysen lagen Tests zugrunde, die Inhalte der Mittelstufe (Klasse 10) und der gymnasialen Oberstufe (Klasse 12) berücksichtigten. In der Gesamtstichprobe zeigte sich ein Zuwachs, der einem $d=$ 1,11 entsprach. In Leistungskursen lag nach Kontrolle des Vorwissens der Zuwachs um 0,6 SD höher als in Grundkursen. Die Zuwächse waren vor allem bezüglich der Inhalte der Sekundarstufe I sichtbar. Dies deckt sich mit dem fachspezifischen Auftrag der gymnasialen Oberstufe, in der unter anderem in Mathematik eine vertiefte Allgemeinbildung erworben werden soll, auch bezogen auf die bereits früher erworbenen Fähigkeiten und Fertigkeiten (vgl. KMK 2012). Insgesamt sprechen die Befunde also dafür, dass es in der gymnasialen Oberstufe auch mit Bezug zu Inhalten der Sekundarstufe I zu einem Anstieg der Kompetenzen kommt.

Retelsdorf et al. (2017) sind für das Fach Mathematik der Frage nachgegangen, welche Kompetenzniveaus auf der Skala der Bildungsstandards Auszubildende (Biologie- und Chemielaborantin bzw. -laborant, $n=632$, gewerblich-technische Berufe, $n=1244$; Industriekaufleute, $n=645$ ) im beruflichen Bildungssystem getrennt nach Schulabschlüssen zu Beginn ihrer Ausbildung erreichen. Insgesamt zeigte sich, dass Auszubildende, die vorher das Abitur abgelegt hatten, höhere Kompetenzen auswie-

Tab. 3 Zuwächse in der mathematischen Grundbildung von Beginn der 11. zum Ende der 13. Jahrgangsstufe nach Schulform und Kursniveau (Effektstärke $d$ )

\begin{tabular}{llll}
\hline Schulform & Gesamt & Leistungskurs & Grundkurs \\
\hline Gymnasium & 0,38 & 0,92 & 0,30 \\
Gesamtschule & 0,42 & 1,01 & 0,30 \\
Aufbaugymnasium & 0,30 & 0,61 & 0,26 \\
Technisches Gymnasium & 0,49 & 0,84 & 0,42 \\
Wirtschaftsgymnasium & 0,31 & 0,64 & 0,21 \\
\hline
\end{tabular}

Quelle: Behörde für Schule und Berufsbildung (2012) 
sen als Auszubildende, die ihre Schullaufbahn mit dem MSA abgeschlossen hatten. Im Vergleich zu den Neuntklässlerinnen und -klässlern des IQB-LV 2012 an Gymnasien schnitten die Abiturientinnen und Abiturienten in dieser Studie nicht besser ab. Für diese Ergebnisse sind zwei Interpretationen denkbar. Zum einen könnten Abiturientinnen und Abiturienten, die zunächst eine Ausbildung anstelle eines Studiums beginnen, geringe Kompetenzen im Vergleich zu ihren Mitschülerinnen und Mitschülern aufweisen. Zum anderen könnte in der gymnasialen Oberstufe der Stoff der Sekundarstufe I eher gehalten aber nicht vertieft worden sein. Gegen letztere Interpretation sprechen allerdings die oben referierten Befunde zu Leistungsveränderungen in der Oberstufe.

\section{Fragestellungen}

In verschiedenen empirischen Untersuchungen konnte gezeigt werden, dass viele Schülerinnen und Schüler am Ende der gymnasialen Oberstufe die Ziele voruniversitären Mathematikunterrichts nicht erreichen. Teilweise wurden nicht einmal die Erwartungen der Sekundarstufe I erfüllt, weiterhin zeigten sich besondere Defizite, wenn das Abitur nicht am allgemeinbildenden Gymnasium erworben wurde. Auf der anderen Seite weisen die wenigen längsschnittlichen Studien darauf hin, dass im Verlauf der gymnasialen Oberstufe erhebliche Zuwächse in den voruniversitären Kompetenzen und denen der Sekundarstufe I zu verzeichnen sind (Köller et al. 1999). Vor allem Schülerinnen und Schüler, die auf erhöhtem Anforderungsniveau mit entsprechender Wochenstundenzahl unterrichtet werden, steigern ihre Kompetenzen erheblich. Hinzu kommt für den Vergleich zwischen dem IQB-LV 2012 (9. Klasse) und dem Erreichen der standardbezogenen Kompetenzen am Ende der gymnasialen Oberstufe ein positiver Selektionsprozess beim Übergang in die Sekundarstufe II. Hinsichtlich der Profiloberstufe in der gymnasialen Oberstufe in Schleswig-Holstein lassen sich daraus folgende Vorhersagen für die Mathematikkompetenzen am Ende der Qualifizierungsphase ableiten:

1. An allgemeinbildenden Gymnasien, in denen Mathematik auf erhöhtem Anforderungsniveau unterrichtet wird, zeigen sich am Ende der gymnasialen Oberstufe deutlich höhere mathematische Kompetenzen als in der schleswig-holsteinischen Vergleichsgruppe der Sekundarstufe I. Wir erwarten einen Unterschied, der mindestens einer Effektstärke von Cohens $d=0,50$ entspricht.

2. Die Erwartungen der Standards für den MSA (Kompetenzstufe III oder höher) werden dementsprechend von sehr großen Teilen der Abiturientinnen und Abiturienten erreicht. Wir erwarten aufgrund der vermuteten mittleren Zuwächse, dass alle Schülerinnen und Schüler den Regelstandard des MSA erreichen.

3. Die Profile an allgemeinbildenden Gymnasien unterscheiden sich erheblich; die mathematischen Kompetenzen in naturwissenschaftlichen Profilen liegen signifikant über denen der übrigen Profile. Aufgrund der unterschiedlichen Lerngelegenheiten und der Selektivität bezogen auf die Profile erwarten wir auch bezogen auf diesen Vergleich Unterschiede, die mindestens einer Effektstärke von Cohens $d=$ 0,50 entsprechen. 
4. Die mathematischen Kompetenzen in den unterschiedlichen Zweigen des beruflichen Gymnasiums fallen deutlich schwächer aus als an allgemeinbildenden Gymnasien.

5. Im technischen Zweig, in dem substanzielle Teile der Schülerinnen und Schüler an beruflichen Gymnasien auf erhöhtem Anforderungsniveau unterrichtet werden, liegen die standardbezogenen Mathematikkompetenzen über denen, die am Ende der 9. Jahrgangsstufe in allgemeinbildenden Gymnasien in Schleswig-Holstein erreicht wurden. Aufgrund der unterschiedlichen Lerngelegenheiten und der Selektivität bezogen auf die Zweige erwarten wir bezogen auf diesen Vergleich Unterschiede, die mindestens einer Effektstärke von Cohens $d=0,50$ entsprechen.

\section{Methode}

Die Anlage und Durchführung der LISA 6-Studie ist detailliert in Leucht et al. (2016) beschrieben. Wir beschränken uns daher im Folgenden auf wenige Informationen. Ausführlicher wird eine Linking-Studie vorgestellt, in der die Mathematikitems aus LISA 6 mit Items aus dem IQB-LV 2012 (Pant et al. 2013) auf einer gemeinsamen Metrik skaliert wurden.

\subsection{Durchführung und Stichprobe der LISA 6-Studie}

In Schleswig-Holstein ist die Oberstufe an allgemeinbildenden Gymnasien seit dem Schuljahr 2008/09 als Profilsystem strukturiert. In beruflichen Gymnasien wird weiterhin in Grund- und Leistungskursen unterrichtet, wobei einer der Leistungskurse die inhaltliche Schwerpunktsetzung des jeweiligen Zweiges abbildet. An der LISA 6-Studie nahmen im Frühjahr $2013 N=3775$ Abiturientinnen und Abiturienten der 13. Jahrgangsstufe aus allen Profilen und Zweigen teil. An den allgemeinbildenden Gymnasien wurde eine Stichprobe gezogen, während für die beruflichen Gymnasien eine Vollerhebung stattfand. Die Verteilung der Schülerinnen und Schüler auf die Profile sowie auf die Zweige ist in Tab. 4 abgebildet.

Um die Repräsentativität der Ergebnisse gewährleisten zu können, wurden die Daten unter Zuhilfenahme von Fallgewichten analysiert. Die Testungen fanden an einem Schultag statt und dauerten fünf Zeitstunden. Die Schülerinnen und Schüler beantworten im Testteil 2 einen Mathematik- und einen Naturwissenschaftstest von jeweils 30 Minuten. Im Testteil 1 wurden Kompetenzen in Englisch untersucht und im Testteil 3 wurden kognitive Grundfähigkeiten getestet sowie ein Fragebogen beantwortet.

\subsection{Der Mathematiktest}

Die Schülerinnen und Schüler bearbeiteten den NEPS-Studierendentest, welcher mathematische Kompetenzen von Studienanfängerinnen und -anfängern beliebiger Fächer erfassen soll (Ehmke et al. 2009). Der Test umfasst 20 Multiple-choice Items. Innerhalb des NEPS wird mathematische Kompetenz in einem Kompetenzstrukturmodell beschrieben, welches eine inhaltliche und eine prozessbezogene Komponente 
Tab. 4 Verteilung der Schülerinnen und Schüler auf die Profile an allgemeinbildenden und Zweige an beruflichen Gymnasien (Angaben für auf Populationsebene gewichtete Daten)

\begin{tabular}{llll}
\hline Profil & Gesamt ungewichtet & Gesamt (gewichtet) & \% weiblich \\
\hline Naturwissenschaftlich & 452 & 2377 & 38,3 \\
Sprachlich & 276 & 1476 & 82,2 \\
Gesellschaftswissenschaftlich & 430 & 2314 & 36,4 \\
Ästhetisch & 122 & 739 & 83,8 \\
Sportlich & 80 & 434 & 34,9 \\
Technik & 505 & - & 22,2 \\
Wirtschaft & 920 & - & 53,5 \\
Agrar/Päd/Psy & 77 & - & 80,5 \\
Ernährung & 362 & - & 77,3 \\
Gesundheit & 417 & - & 84,9 \\
\hline
\end{tabular}

Aufgrund der geringen Fallzahlen wurden die Profile Agrarwissenschaft, Pädagogik und Psychologie für alle Analysen zusammengefasst; keine gewichteten Häufigkeiten an beruflichen Gymnasien, da hier eine Vollerhebung durchgeführt wurde

ausdifferenziert. Die inhaltliche Dimension wird in die vier übergreifenden mathematischen Ideen Quantität, Veränderung und Beziehung, Raum und Form sowie Daten und Zufall unterteilt. Die prozessorientierte Dimension wird in sechs kognitive Komponenten mathematischer Denkprozesse unterschieden: Kommunizieren, Modellieren, Problemlösen, Repräsentieren (Darstellungen verwenden) und technische Fertigkeiten einsetzen (Kampa et al. 2016a). In der LISA 6-Studie betrug die EAP/PV Reliabilität des NEPS-Studierendentests 0,66, in der Linking-Studie 0,57. Der NEPS-Studierendentest wurde sowohl in LISA 6 als auch in der Linking-Studie angewendet.

\subsection{Linking-Studie - Durchführung, Stichprobe und Analysen}

Ziel der Verlinkung war es, die mathematischen Kompetenzen der LISA 6-Abiturientinnen und Abiturienten auf dem Kompetenzstufenmodell des IQB für den MSA verorten zu können. Ausgangspunkt für das Linking waren zwei unterschiedliche Stichproben (allgemeinbildende und berufliche Gymnasien in LISA 6 vs. Gymnasien und weitere Schulen der Sekundarstufe I im IQB-LV 2012) aus unterschiedlichen Populationen (13. Jahrgangsstufe vs. 9. Jahrgangsstufe), welche unterschiedliche Kompetenztests bearbeitet hatten (NEPS vs. IQB-LV 2012). Um Skalen miteinander zu verlinken, werden in der Literatur unterschiedliche Linkingmethoden vorgeschlagen (Nissen et al. 2015). Für den vorliegenden Fall wurde ein commonitem nonequivalent groups design angewendet (Kolen und Brennan 2004), wobei zunächst eine Linking-Studie durchgeführt wurde, um die Skalen der beiden Kompetenztests anhand einer gemeinsamen Stichprobe miteinander zu verbinden. An der Linking-Studie nahmen 142 Schülerinnen und Schüler (55\% weiblich) an drei Gymnasien in Schleswig-Holstein am Ende der 12. Jahrgangsstufe teil und bearbeiteten sowohl die NEPS- als auch die MSA-Bildungsstandardaufgaben. Es wurden 56 Bildungsstandard-Items eingesetzt, von denen 22 Multiple-Choice Items sind. 


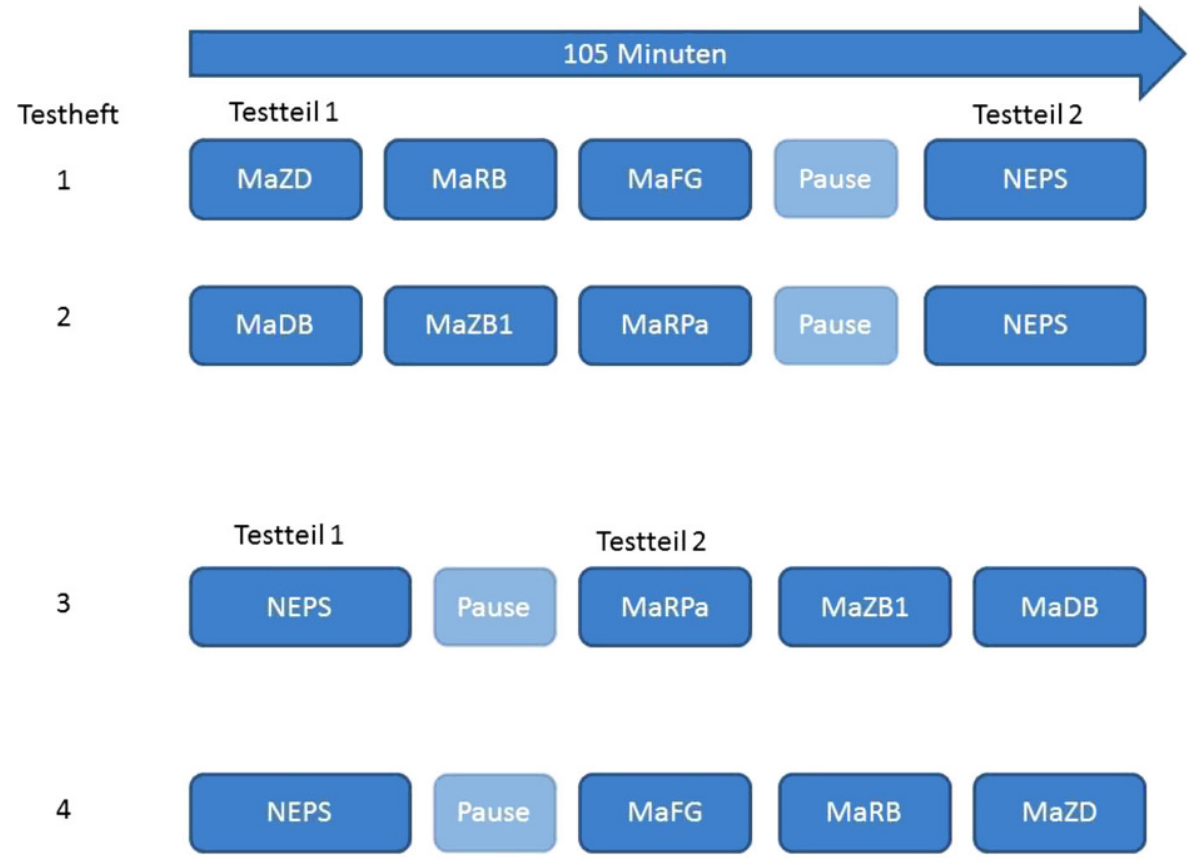

Abb. 2 Testdesign und Ablauf der Testung. Für Bildungsstandardblöcke Buchstabe 1 und 2: MA Mathe, Buchstabe 3: ZZahl, $R$ Raum \& Form, F Funktionaler Zusammenhang, D Daten und Zufall, NEPS National Educational Panel Study, min Minuten

Insgesamt 25 Items hatten ein offenes Antwortformat und 9 Items ein halboffenes Antwortformat.

Die Schülerinnen und Schüler waren mit Ausnahme des sportlichen Profils in allen Profilen der allgemeinbildenden Schulen vertreten. Die standardisierte Testung wurde von geschulten Testleiterinnen durchgeführt und orientierte sich an den Testbedingungen im IQB-Ländervergleich bzw. in LISA 6.

Die Daten wurden mit vier unterschiedlich aufgebauten Testheften erhoben (siehe Abb. 2). Um Positionseffekte zu vermeiden, wurde der NEPS-Studierendentest in den Testheften 1 und 2 an das Ende der Testung gestellt, in den Testheften 3 und 4 an den Anfang. Für die Bearbeitung der NEPS-Aufgaben standen den Schülerinnen und Schülern 30 Minuten zur Verfügung und die Aufgaben wurden von der gesamten Stichprobe bearbeitet.

Da die hohe Anzahl an Mathematikitems zu den Bildungsstandards nicht von allen Schülerinnen und Schülern beantwortet werden konnte, wurden sechs Blöcke aus dem IQB-LV 2012 ausgewählt, deren Bearbeitung jeweils 20 Minuten betrug. Die Reihenfolge der Aufgabenblöcke wurde innerhalb der Testhefte ebenfalls variiert (siehe Abb. 2). Die Testzeit inklusive Pause betrug insgesamt 105 Minuten. Jedes Bildungsstandards-Item wurde im Mittel von 61 Personen bearbeitet $(S D=8,78)$. Die EAP/PV-Reliabilität lag bei 0,82 . 


\subsection{Analysen}

Zur Generierung der Itemparameter und Plausible Values (PVs) wurden die 76 Items mittels eines eindimensionalen 1PL-Modells in ConQuest (Wu et al. 2007) mit einem Hintergrundmodell (Geschlecht und Schule) skaliert. In dieser Skalierung wurden die Itemparameter der 56 Bildungsstandards-Items auf die Werte aus dem IQBLV 2012 fixiert und die auf dieser Grundlage geschätzten NEPS-Itemparameter ausgelesen. Somit wurden die Itemparameter der NEPS-Items auf die nationale Metrik der Bildungsstandards in der 9. Jahrgangsstufe projiziert.

Die aus der Skalierung der Linking-Studie generierten NEPS-Itemparameter konnten im zweiten Schritt auf den LISA 6-Datensatz angewendet werden. Mit dem in LISA 6 verwendeten Hintergrundmodell (siehe Leucht und Köller 2016) wurde eine eindimensionale Skalierung der LISA 6-Stichprobe durchgeführt, wobei die NEPS-Items auf die Itemparameter aus der Linking-Studie fixiert wurden. So wurden die Personenparameter (fünf PVs pro Person) auf der nationalen Metrik der Bildungsstandards ( $M=500 ; S D=100$ in der 9. Jahrgangsstufe) geschätzt und in den weiteren Analysen verwendet.

Alle Analysen zur Beantwortung der Fragestellungen wurden wie in LISA 6 mit 3461 Schülerinnen und Schülern durchgeführt. Hierbei handelt es sich um die Schülerinnen und Schüler, die valide Angaben zu ihren Wochenstunden in Mathematik gemacht hatten (siehe Kampa et al. 2016a). Zur Bestimmung der Kompetenzen im Vergleich zur Sekundarstufe I haben wir zunächst in Mplus (Muthén und Muthén 1998-2012) die mittleren Kompetenzwerte der LISA 6-Schülerinnen und Schüler auf der Bildungsstandards-Metrik berechnet ${ }^{1}$. In einem weiteren Schritt haben wir, wiederum mit den Kompetenzstufengrenzen aus dem IQB-LV 2012, die Verteilung der Schülerinnen und Schüler auf den Kompetenzstufen in SPSS Version 22 (IBM 2013) berechnet. Beide Berechnungen (Mittelwerte und Kompetenzstufenverteilung) wurden für die gesamte Stichprobe sowie getrennt nach Gymnasialtyp und nach Profilen bzw. Zweigen durchgeführt. Für die Verteilungsanalysen wurden in einem ersten Schritt die Kompetenzstufenverteilung für jede Schülerin bzw. jeden Schüler dummykodiert und in einem zweiten Schritt die Analysen für die fünf PVs in Mplus integriert.

\section{Ergebnisse}

Die Abiturientinnen und Abiturienten in Schleswig-Holstein erreichen im Mittel 679 Kompetenzpunkte $(S D=58,63 S E=3,09)$ in Mathematik. Sie liegen damit im Vergleich zu den Ergebnissen des IQB-LV 2012 ca. 92 Kompetenzpunkte höher als die vergleichbare Population (Gymnasiastinnen und Gymnasiasten am Ende der Sekundarstufe I in Schleswig-Holstein). Dies entspricht einer mittleren Verbesserung in den Kompetenzen von 23 Kompetenzpunkten pro Schuljahr, welche einer Effektstärke von $d=1,58$ entspricht. Somit kann die Hypothese $1-$ auch bezogen auf die erwartete Effektstärke - gestützt werden. In den allgemeinbildenden Gym-

\footnotetext{
1 Mplus bietet die Möglichkeit, die PVs für Analysen zu Punktschätzern zu integrieren.
} 


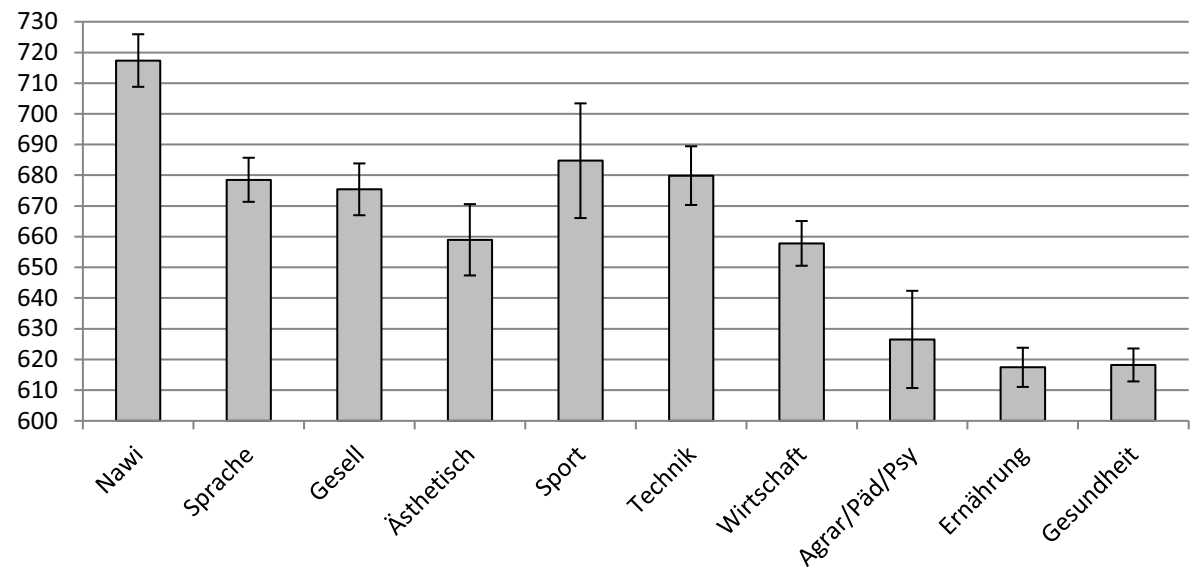

Abb. 3 Standardbezogene Kompetenzunterschiede (Mittelwerte und 95\%-Vertrauensintervalle) in Mathematik bezogen auf die Profile in allgemeinbildenden und die Zweige in beruflichen Gymnasien. Nawi Naturwissenschaftlich, Gesell Gesellschaftswissenschaftlich, Agrar/Päd/Psy Agrarwissenschaft, Pädagogik und Psychologie

nasien erreichen die Schülerinnen und Schüler 689 Kompetenzpunkte $(S D=54,24$ $S E=3,55)$ während sie in beruflichen Gymnasien 648 Kompetenzpunkte $(S D=$ $57,33 S E=2,94$ ) erreichen. Dieser Unterschied beläuft sich auf $d=0,75$. Die Schülerinnen und Schüler an den allgemeinbildenden Gymnasien zeigen am Ende der Oberstufe somit deutlich höhere mathematische Kompetenzen als die Jugendlichen am Ende der Sekundarstufe I (Hypothese 1) und als die Jugendlichen in den beruflichen Gymnasien (Hypothese 4). Es ist jedoch auch in den beruflichen Gymnasien ein deutlicher Kompetenzvorsprung im Vergleich zum Ende der Sekundarstufe I zu vermerken. Während Abb. 3 die entsprechenden Mittelwertunterschiede zwischen den Profilen bzw. den Zweigen aufzeigt, sind in Tab. 5 die Effektstärken für diese Unterschiede dargestellt.

Es wird deutlich, dass die Schülerinnen und Schüler im naturwissenschaftlichen Profil an allgemeinbildenden Gymnasien die besten Ergebnisse erzielen. Sie liegen mit 717 Kompetenzpunkten zwischen 35 und 42 Kompetenzpunkte vor den Schülerinnen und Schülern im sprachlichen $(d=0,59)$, gesellschaftlichen $(d=0,81)$, ästhetischen $(d=1,13)$ und dem sportlichen Profil $(d=0,63)$ im allgemeinbildenden Gymnasium bzw. im Zweig Technik im beruflichen Gymnasium $(d=0,70)$. Im Vergleich mit den anderen Profilen liegen die Effektstärken durchgängig über der von uns gesetzten Grenze von $d=0,50$ (Hypothese 3 ). Somit sind auch innerhalb der allgemeinbildenden Gymnasien starke Differenzen aufgrund des Wahlverhaltens zu Beginn der Oberstufe zu finden. Weitaus größere Unterschiede zeigen sich im Vergleich der Profile an allgemeinbildenden Gymnasien mit den Zweigen in beruflichen Gymnasien (Hypothese 4). Der größte Abstand über die beiden Schulformen ist zwischen dem naturwissenschaftlichen Profil auf der einen und den Zweigen Ernährung $(d=1,94)$ und Gesundheit $(d=2,01)$ auf der anderen Seite auszumachen. Hier liegt der Unterschied bei ca. 100 Kompetenzpunkten. Schülerinnen und Schüler im Zweig Technik erreichen vergleichbare Kompetenzen wie im sprachlichen, 
Tab. 5 Effektstärken für die Unterschiede zwischen den Profilen an allgemeinbildenden Gymnasien sowie den Zweigen an den beruflichen Gymnasien

\begin{tabular}{llllllllll}
\hline Profil & $(1)$ & $(2)$ & $(3)$ & $(4)$ & $(5)$ & $(6)$ & (7) & (8) & $(9)$ \\
\hline $\begin{array}{l}\text { Naturwissenschaftlich } \\
\text { (1) }\end{array}$ & - & - & - & - & - & - & - & - & - \\
Sprachlich (2) & 0,59 & - & - & - & - & - & - & - & - \\
Gesellschafts- & 0,81 & 0,25 & - & - & - & - & - & - & - \\
wissenschaftlich & & & & & & & & & \\
(3) & & & & & & & & & \\
Ästhetisch (4) & 1,13 & 0,61 & 0,33 & - & - & - & - & - & - \\
Sportlich (5) & 0,63 & 0,06 & 0,18 & 0,53 & - & - & - & - & - \\
Technik (6) & 0,70 & 0,15 & 0,08 & 0,39 & 0,09 & - & - & - & - \\
Wirtschaft (7) & 1,11 & 0,57 & 0,33 & 0,02 & 0,50 & 0,41 & - & - & - \\
Agrar/Päd/Psy (8) & 1,75 & 1,30 & 0,97 & 0,68 & 1,19 & 0,99 & 0,58 & - & - \\
Ernährung (9) & 1,94 & 1,44 & 1,15 & 0,84 & 1,35 & 1,18 & 0,76 & 0,18 & - \\
Gesundheit (10) & 2,01 & 1,51 & 1,19 & 0,89 & 1,44 & 1,22 & 0,77 & 0,18 & 0,02 \\
\hline
\end{tabular}

gesellschaftlichen und sportlichen Profil (Effektstärken zwischen $d=0,08$ und $d=$ 0,09); Schülerinnen und Schüler im Zweig Wirtschaft wie im ästhetischen Profil $(d=$ 0,02). Die weiteren Zweige zeigen keine vergleichbaren Kompetenzausprägungen zu den Profilen an allgemeinbildenden Gymnasien. Die Schülerinnen und Schüler im Zweig Technik zeigen nicht nur die höchsten Kompetenzwerte innerhalb der beruflichen Gymnasialzweige, sie zeigen auch einen deutlichen Vorsprung im Vergleich zur Referenzgruppe in der Sekundarstufe I in Schleswig-Holstein $(d=1,54$ Hypothese 5). Trotz eines deutlichen Vorsprungs der allgemeinbildenden Gymnasien gegenüber den beruflichen Gymnasien gibt es somit einige Zweige an beruflichen Gymnasien, in denen es gelingt, Kompetenzen vergleichbar mit denen in Profilen in allgemeinbildenden Gymnasien zu erzielen. Nicht alle Vergleiche zwischen den Zweigen und den Profilen stützen somit unsere Hypothese 4.

$\mathrm{Zu}$ welcher Verteilung auf die Kompetenzstufen die bereits beschriebenen mittleren Unterschiede am Ende der Abiturphase führen und welche Folgen diese Unterschiede für die Erreichung des Regelstandards zwischen den Profilen bzw. Zweigen haben, berichten wir im Folgenden.

\subsection{Verteilung auf die Kompetenzstufen}

Bezogen auf die Verteilung aller Schülerinnen und Schüler auf die Kompetenzstufen für den MSA befinden sich nur noch 0,2\% der Schülerinnen und Schüler auf der Kompetenzstufe II, 7,0\% auf der Kompetenzstufe III, 40,3\% auf der Kompetenzstufe IV und 52,4\% auf der Kompetenzstufe V. Damit erreichen nahezu alle Schülerinnen und Schüler mindestens den Regelstandard (Kompetenzstufe III) und etwas mehr als die Hälfte sogar den Optimalstandard (Kompetenzstufe V; Hypothese 2). Betrachtet man die Verteilung getrennt nach Schulformen, so befinden sich alle Schülerinnen und Schüler der allgemeinbildenden Gymnasien mindestens auf Kompetenzstufe III (siehe Tab. 6). Auch in den beruflichen Gymnasien verfehlt nur $1 \%$ der Schülerschaft den Regelstandard des MSA. 
Tab. 6 Verteilung der Schülerinnen und Schüler auf die Kompetenzstufen des MSA-Kompetenzstufenmodells für Mathematik nach Schulform (Angaben in Prozent)

\begin{tabular}{llllll}
\hline Schulform & I & II & III & IV & V \\
\hline Allgemeinbildend & - & - & 4,0 & 37,1 & 58,9 \\
Beruflich & - & 1,0 & 16,8 & 50,6 & 31,6 \\
\hline
\end{tabular}

- = keine Schülerinnen und Schüler auf dieser Kompetenzstufe

Tab. 7 Verteilung der Schülerinnen und Schüler auf die Kompetenzstufen des MSA-Kompetenzstufenmodells für Mathematik getrennt nach Profilen bzw. Zweigen (Angaben in Prozent)

\begin{tabular}{llllll}
\hline Profil/Zweig & \multicolumn{3}{l}{ Kompetenzstufe } & & \\
& I & $\begin{array}{l}\text { II Mindest- } \\
\text { standard }\end{array}$ & $\begin{array}{l}\text { III Regel- } \\
\text { standard }\end{array}$ & $\begin{array}{l}\text { IV Regel- } \\
\text { standard + }\end{array}$ & $\begin{array}{l}\text { V Optimal- } \\
\text { standard }\end{array}$ \\
\hline $\begin{array}{l}\text { Naturwissenschaftlich } \\
\text { Sprachlich }\end{array}$ & 0,0 & 0,0 & 0,5 & 22,5 & 77,0 \\
$\begin{array}{l}\text { Gesellschafts- } \\
\text { wissenschaftlich }\end{array}$ & 0,0 & 0,0 & 4,3 & 42,6 & 53,1 \\
Ästhetisch & 0,0 & 0,0 & 5,9 & 43,5 & 50,6 \\
Sportlich & 0,0 & 0,0 & 9,0 & 53,3 & 37,7 \\
Technik & 0,0 & 0,0 & 3,6 & 36,6 & 59,7 \\
Wirtschaft & 0,0 & 0,4 & 6,6 & 36,7 & 56,3 \\
Agrar/Päd/Psy & 0,0 & 0,6 & 10,9 & 51,7 & 36,8 \\
Ernährung & 0,0 & 1,9 & 24,4 & 56,6 & 17,4 \\
Gesundheit & 0,0 & 2,0 & 30,9 & 54,3 & 12,8 \\
\hline Agrar/Pad/Psy Zu, & 0,2 & 1,3 & 28,4 & 60,9 & 9,3 \\
\hline
\end{tabular}

Agrar/Päd/Psy Zusammenschließung der Zweige Agrarwissenschaft, Pädagogik und Psychologie aufgrund geringer Substichprobengröße

Nichtsdestotrotz unterscheidet sich die Verteilung in den beiden Schulformen. Während in den beruflichen Gymnasien ca. die Hälfte der Schülerinnen und Schüler die Kompetenzstufe IV (Regelstandard plus) und weitere ca. $30 \%$ die Kompetenzstufe V (Optimalstandard) erreichen, lässt sich in den allgemeinbildenden Gymnasien ein umgekehrtes Bild erkennen. Hier gelingt es fast $40 \%$ der Schülerschaft mindestens den Regelstandard plus und ca. $60 \%$ der Schülerschaft den Optimalstandard zu erreichen. Weitere Unterschiede lassen sich wiederum zwischen den Profilen und Zweigen finden (siehe Tab. 7).

In beiden Schulformen zeigen sich auch für die Profile bzw. Zweige starke Unterschiede in der Erreichung der standardbezogenen Erwartungen. Im naturwissenschaftlichen Profil erreichen im Prinzip alle Schülerinnen und Schüler mindestens den Regelstandard plus und fast $80 \%$ den Optimalstandard. Während im ästhetischen Profil knapp 10\% nur den Regelstandard erreichen, sind es bei den Mitschülerinnen und -schülern im sprachlichen, gesellschaftswissenschaftlichen und sportlichen Profil ca. $5 \%$. Dementsprechend erreicht die Schülerschaft in diesen Profilen nur zu etwas mehr als $50 \%$ den Optimalstandard, während es im ästhetischen Profil nur knapp $40 \%$ sind. In den Zweigen an beruflichen Gymnasien werden generell geringere Kompetenzstände erreicht. So erreichen nur ca. $10 \%$ der Schülerinnen und Schüler in den Zweigen Ernährung und Gesundheit den Optimalstandard, im Zweig 
Technik hingegen über $50 \%$. Ein beachtlicher Teil der Schülerschaft in den nichttechnischen Zweigen an beruflichen Gymnasien erreicht nur den Regelstandard des MSA (zwischen 10,9\% im Zweig Wirtschaft und 30,9\% im Zweig Ernährung).

\section{Diskussion}

Die Diskussion um Erträge der gymnasialen Oberstufe wird seit Erscheinen der TIMSS-Ergebnisse (Baumert et al. 2000a, 2000b) geführt. Wiederholt wurde gezeigt (zuletzt Kampa et al. 2016a), dass große Anteile der Abiturientinnen und Abiturienten im Fach Mathematik Ziele des voruniversitären Unterrichts nicht erreichen. Umso dringlicher stellte sich die Frage, ob zumindest im Sinne eines Mindeststandards gymnasialer Bildung die Erwartungen erfüllt werden, die mit der Vergabe des MSA verknüpft sind. Genau hier setzten unsere Analysen an, indem Kompetenzen im Fach Mathematik am Ende der gymnasialen Oberstufe auf der nationalen Metrik für den MSA berichtet und mit den Ergebnissen aus dem IQB-LV 2012 aus Schleswig-Holstein (vgl. Pant et al. 2013) verglichen wurden.

Zwischen den Stichproben des IQB-LV 2012 und LISA 6 lagen vier Schuljahre (Ende der 9. vs. Ende der 13. Jahrgangsstufe). Für diese Zeitdifferenz konnten wir mit unserer Linking-Studie für die gymnasiale Schülerschaft in Schleswig-Holstein eine standardbezogene Differenz von 92 Kompetenzpunkten verzeichnen. Dies entspricht einer mittleren Differenz von 23 Kompetenzpunkten pro Jahr. Wir diskutieren die Befunde im Folgenden zunächst aus der Perspektive der Ziele voruniversitärer Bildung im Fach Mathematik. Anschließend widmen wir uns den gefundenen Kompetenzdifferenzen zwischen Profilen und Zweigen und enden mit Hinweisen auf Grenzen der hier berichteten Untersuchung und einem Resümee.

\subsection{Einordnung der Befunde mit Bezug auf voruniversitäre Unterrichtsziele}

Obwohl die Kernziele des Faches Mathematik in der gymnasialen Oberstufe in der Vermittlung von voruniversitären Inhalten und Kompetenzen liegen, soll der Unterricht auch auf die Vertiefung der in der Sekundarstufe I erworbenen Kompetenzen abzielen. Dementsprechend heißt es in den Bildungsstandards im Fach Mathematik für die Allgemeine Hochschulreife: „Die Bildungsstandards für die Allgemeine Hochschulreife sind eine direkte und organische Fortführung der Bildungsstandards für den Mittleren Schulabschluss. Die in der Sekundarstufe I erworbenen Kompetenzen sind unverzichtbare Grundlage für die Arbeit in der Sekundarstufe II. Sie werden dort beständig vertieft und erweitert und können damit auch Gegenstand der Abiturprüfung sein“ (KMK 2012, S. 12). Da in empirischen Studien zu Erträgen voruniversitären Lernens die Frage nach der Erreichung von Zielen bezüglich der Inhalte der gymnasialen Oberstufe stand, wurde bislang nur selten untersucht, ob diese Vertiefung gelingt. Die TIMSS-Ergebnisse (Baumert et al. 2000a, 2000b) machten diesbezüglich bereits Defizite deutlich; diese Befundlage hat sich bis heute nicht geändert (vgl. Kampa et al. 2016a). Im Bereich der Ziele der Sekundarstufe I implizierten bisherige Studien, dass rund ein Fünftel der Abiturientinnen und Abiturienten diese selbst am Ende der gymnasialen Oberstufe nicht erreichte (Baumert 
et al. 2000a, 2000b). Empirische Arbeiten mit Bezug zu normativen Erwartungen der Sekundarstufe I fehlten aber. Diese Lücke wird mit dieser Arbeit geschlossen und die Befunde machen deutlich, dass quasi alle Schülerinnen und Schüler der allgemeinbildenden und beruflichen Gymnasien am Ende der gymnasialen Oberstufe die mit den MSA-Bildungsstandards verbundenen Kompetenzen erreichen bzw. diese übertreffen. Der Vergleich der Zahlen aus den Tab. 2 und 5 macht deutlich, dass die LISA 6Schülerinnen und Schüler in ihrer Zuordnung zu den Kompetenzstufen tatsächlich höher liegen als die in Schleswig-Holstein 2012 getesteten Neuntklässlerinnen und -klässler. Geht man davon aus, dass keine systematischen außerschulischen Lerngelegenheiten für Mathematik bestehen (vgl. Köller und Baumert 2012), so scheint der voruniversitäre Unterricht die in der Sekundarstufe I erworbenen Kompetenzen zu vertiefen. Folgt man der Rationale, dass mit dem Erreichen der MSA-Bildungsstandards ein erfolgreicher Übertritt in die berufliche Erstausbildung möglich ist, so kann davon ausgegangen werden, dass quasi alle Abiturientinnen und Abiturienten Schleswig-Holsteins die Schule mit mathematischen Kompetenzausprägungen verlassen, die eine erfolgreiche Einfädelung in die duale Ausbildung - auch in mathematikintensiven, kaufmännischen Berufen - erlauben. Zugegebenermaßen besteht hierin nicht das Kernziel voruniversitären Lernens. Grenzen haben diese Erörterungen darin, dass wir keine Längsschnittdaten analysieren konnten. Auf der anderen Seite fügen sich die Befunde in die ermittelten Zuwächse bisheriger Studien ein (Köller et al. 1999; Lee 2010; Behörde für Schule und Berufsbildung 2012).

\subsection{Unterschiede zwischen Profilen und Zweigen}

Bereits in früheren Publikationen (Kampa et al. 2016a, 2016b) sind wir vertiefend auf die Kompetenzunterschiede in Mathematik zwischen Profilen und Zweigen eingegangen, dort allerdings mit Blick auf die voruniversitären Kompetenzen. Bemerkenswert ist insbesondere die Größe der von uns aufgedeckten Unterschiede. Der kleinste Unterschied lag bereits bei 33 Kompetenzpunkten (naturwissenschaftliches vs. sportliches Profil). Damit beträgt bereits der kleinste Unterschied ein Drittel einer Standardabweichung und entspricht damit einem Zuwachs von rund einem Schuljahr in der gymnasialen Oberstufe. Der größte Unterschied lag bei 100 Punkten (naturwissenschaftliches Profil vs. Zweig Ernährung) und entspricht einer ganzen Standardabweichung und damit ungefähr einem Zuwachs, der in der gesamten Oberstufe erwartet werden könnte. Wie bereits im TOSCA-Projekt aufgezeigt wurde (Watermann et al. 2004), konnten auch wir belegen, dass es in den beruflichen Gymnasien nur Schülerinnen und Schülern im Zweig Technik gelingt, ähnliche Kompetenzen wie an allgemeinbildenden Gymnasien zu erreichen.

Hinsichtlich der Unterschiede zwischen beruflichen und allgemeinbildenden Gymnasien könnten die Unterschiede in erheblichem Maße auf Differenzen in den Eingangsvoraussetzungen zurückzuführen sein. So zeigten frühere Analysen, dass große Unterschiede zwischen beiden Gruppen in den kognitiven Grundfähigkeiten bestehen (Köller 2016a). Auch konnte bereits im IQB-LV 2012 für das Fach Mathematik gezeigt werden, dass in den Gymnasien deutlich höhere Kompetenzen erreicht werden als in den übrigen Schulformen (Pant et al. 2013). Die beruflichen Gymnasien in Schleswig-Holstein attrahieren insbesondere Realschülerinnen und 
-schüler, die das Abitur über das berufliche Gymnasium anstreben (Köller 2016a). Die von uns gefundenen Unterschiede zwischen den Schulformen werden somit zu einem erheblichen Anteil durch Eingangsmerkmale verursacht sein. Differenzen im Vorwissen dürften auch eine zentrale Quelle für die Unterschiede zwischen Profilen innerhalb von allgemeinbildenden und zwischen Zweigen innerhalb von beruflichen Gymnasien sein (s. hierzu auch Köller 2016b sowie Kampa et al. 2016a, 2016b). Im Zweig Technik wählen z. B. große Zahlen von Schülerinnen und Schülern einen mathematischen oder naturwissenschaftlichen Leistungskurs. Die damit verbundene höhere Stundenzahl ebenso wie die stärkere Vertiefung der Themen dürften Differenzen zwischen den Zweigen erhöhen.

In allgemeinbildenden Gymnasien dagegen werden alle Schülerinnen und Schüler vierstündig auf erhöhtem Anforderungsniveau unterrichtet. Aber auch hier bestehen Unterschiede in der Eingangsselektivität (höhere kognitive Grundfähigkeiten in den naturwissenschaftlichen Profilen; Köller 2016b). Diese Unterschiede aufgrund der Eingangsvoraussetzungen könnten während der Abiturphase durch weitere Mechanismen verstärkt werden. Da der Unterricht in den meisten Fällen im Profilverband stattfindet, können unterschiedliche Erwartungshaltungen und Vergleichsprozesse vermutet werden. Die Lehrkräfte könnten die höchsten Erwartungen an die Schülerinnen und Schüler im naturwissenschaftlichen Profil stellen, welche wiederum auch die höchsten Erwartungen an sich und ihren Klassenverband stellen. Gleiche Prozesse in die entgegengesetzte Richtung sind in den nicht-naturwissenschaftlichen Profilen zu erwarten. Die Lehrkräfte könnten den Unterricht aufgrund ihrer Erwartungen an die jeweilige Schülerschaft anpassen und die Schülerinnen und Schüler werden durch die beschriebene Wahrnehmung des Klassenverbandes geprägt. Die skizzierten Mechanismen wurden bisher nicht untersucht und sollten Gegenstand zukünftiger Untersuchungen sein.

\subsection{Verteilung auf die Kompetenzstufen}

Bezogen auf die Kompetenzstufenverteilung konnten wir zeigen, dass sowohl im allgemeinbildenden als auch im beruflichen Gymnasium so gut wie alle Schülerinnen und Schüler mindestens die Kompetenzstufe III und damit den Regelstandard für den MSA erreichen. Trotz der im Mittel unterschiedlichen Kompetenzen zwischen den Schulformen erreicht somit die gesamte Schülerschaft diesen normativ festgelegten Standard. Es bleibt jedoch anzumerken, dass es sich um Erwartungen an die Leistungen am Ende der Sekundarstufe I handelt, die anscheinend erst am Ende der Sekundarstufe II erreicht werden. Den Optimalstandard, welcher eher als ein zu erreichendes Ziel am Ende der Abiturphase anzusehen sein könnte, erreichen rund $60 \%$ der Schülerinnen und Schüler am allgemeinbildenden und lediglich rund $30 \%$ der Schülerschaft an beruflichen Gymnasien. Dieser Prozentsatz von Schülerinnen und Schülern, die die Erwartungen der gymnasialen Oberstufe nicht erfüllen, deckt sich auch mit den bereits hierzu publizierten Studien (siehe z. B. Köller et al. 2004; Kampa et al. 2016a).

Konsequenterweise erreichen im Prinzip auch alle Schülerinnen und Schüler der unterschiedlichen Profile bzw. Zweige mindestens die Kompetenzstufe III - den Regelstandard des MSA. Innerhalb der Verteilung auf die Kompetenzstufen III, IV 
und V konnten wir jedoch erhebliche Unterschiede aufzeigen. Die Erreichung des Optimalstandards liegt zwischen $77 \%$ der Schülerinnen und Schüler im naturwissenschaftlichen Profil und 9\% im Zweig Gesundheit. Auch an dieser Stelle stellt sich folglich die weitestgehend bildungspolitisch-normative Frage, welche mathematischen Kompetenzen die Schülerinnen und Schüler in den Profilen bzw. Zweigen über den Regelstandard des MSA hinaus erfüllen sollen.

\subsection{Limitationen und Konklusion}

Mit der vorgestellten Studie konnten wir zwar neue Einblicke in die Leistungsfähigkeit der gymnasialen Oberstufe generieren, jedoch sollten ebenfalls einschränkende Aspekte der Studie berücksichtigt werden. Zum einen haben wir keine Längsschnittstudie durchgeführt. Um die Kompetenzzuwächse zu modellieren, die tatsächlich auf die bereitgestellten Lerngelegenheiten in der Abiturphase zurückzuführen sind, bedarf es in der zukünftigen Forschung Längsschnittstudien in dieser Phase der Beschulung, welche dezidiert die Erreichung von Bildungsstandards im Fokus hat. Des Weiteren lässt sich die relativ kleine Stichprobe von 142 Schülerinnen und Schülern in unserer Linking-Studie anführen. Zwar hatten wir hinreichende Zahlen von Schülerinnen und Schülern pro Item zur Verfügung, um robuste Itemparameter zu schätzen, in Studien mit ähnlicher Ausrichtung könnte die Stichprobe jedoch zur Sicherung kleinerer Standardfehler der Itemparameter und damit auch zur Reduzierung von Linkingfehlern erhöht werden. Darüber hinaus beschränkt sich die Studie auf Kompetenzen in Mathematik. Eine weiterführende Betrachtung anderer Kompetenzbereiche, die in den LSA erhoben werden, erscheint ebenfalls sinnvoll.

Wir möchten unterstreichen, dass es sich bei den MSA-Bildungsstandards Mathematik um normative Erwartungen handelt, die drei Jahre vor dem Abitur erreicht werden sollten. Insofern haben wir - motiviert durch die niedrigen Kompetenzen von Abiturientinnen und Abiturienten bei Oberstufenaufgaben - in unserer Arbeit die Zielmarke mathematischen Lernens in der gymnasialen Oberstufe sehr niedrig definiert. Unsere positiven Ergebnisse sind somit kein Beitrag zur Evaluierung der voruniversitären Mathematik der Schülerinnen und Schüler, welches eines der zentralen Ziele der Oberstufe darstellt. Tatsächlich beleuchten wir, dass ein Missstand, der am Ende der Sekundarstufe I aufgezeigt werden konnte, innerhalb der Abiturphase weitestgehend behoben werden konnte. Etwaige weitere Zielmarken für den Abschluss nach Durchlaufen der Sekundarstufe II sollten Thema weiterer Untersuchungen sein.

Eine weitere Beschränkung ist, dass wir wenig über das konkrete Unterrichtsgeschehen im Mathematikunterricht der gymnasialen Oberstufe wissen. Inwieweit der Unterricht tatsächlich die Vertiefung der Inhalte der Sekundarstufe I zulässt und wie stark diese Lerngelegenheiten zwischen Zweigen bzw. Profilen variieren, muss in zukünftigen Forschungsarbeiten geklärt werden. Festzuhalten bleibt aber, dass junge Erwachsene, die eine gymnasiale Oberstufe mit der Hochschulzugangsberechtigung verlassen, ein hinreichendes Maß an mathematischer Grundbildung erreichen - sofern man das Erreichen der Standards für den MSA akzeptiert.

Frühere Auswertungen hatten gezeigt (Kampa et al. 2016a, 2016b), dass in der Beherrschung der voruniversitären mathematischen Kompetenzen - eine wichtige 
Voraussetzung zur Bewältigung mathematikintensiver Studienfächer - erhebliche Schwächen zu verzeichnen sind. Unsere Befunde zeigen demgegenüber, dass nahezu der gesamte Abiturjahrgang die Schule mit mathematischen Kompetenzen verlässt, die für einen erfolgreichen Umgang mit mathematischen Kontexten im Alltag und eine hinreichende Voraussetzung für eine erfolgreiche berufliche Erstausbildung (auch in mathematikintensiven Berufen) stehen dürften.

Open Access Dieser Artikel wird unter der Creative Commons Namensnennung 4.0 International Lizenz (http://creativecommons.org/licenses/by/4.0/deed.de) veröffentlicht, welche die Nutzung, Vervielfältigung, Bearbeitung, Verbreitung und Wiedergabe in jeglichem Medium und Format erlaubt, sofern Sie den/die ursprünglichen Autor(en) und die Quelle ordnungsgemäß nennen, einen Link zur Creative Commons Lizenz beifügen und angeben, ob Änderungen vorgenommen wurden.

\section{Literatur}

Baumert, J., Bos, W., \& Lehmann, R. (2000a). TIMSS/III: Dritte Internationale Mathematik- und Naturwissenschaftstudie. Mathematische und naturwissenschaftliche Bildung am Ende der Schullaufbahn. Bd. 1: Mathematische und naturwissenschaftliche Grundbildung am Ende der Pflichtschulzeit. Opladen: Leske + Budrich.

Baumert, J., Bos, W., \& Lehmann, R. (2000b). TIMSS/III: Dritte Internationale Mathematik- und Naturwissenschaftstudie. Mathematische und naturwissenschaftliche Bildung am Ende der Schullaufbahn. Bd. 2: Mathematische und physikalische Kompetenzen am Ende der gymnasialen Oberstufe. Opladen: Leske + Budrich.

Behörde für Schule und Berufsbildung (2012). LAU - Aspekte der Lernausgangslage und der Lernentwicklung. Klassenstufen 11 und 13. Münster: Waxmann.

Blum, W. (2006). Einführung. In W. Blum, C. Drüke-Noe, R. Hartung, \& O. Köller (Hrsg.), Bildungsstandards Mathematik: konkret. Sekundarstufe I: Aufgabenbeispiele, Unterrichtsanregungen, Fortbildungideen (S. 14-32). Berlin: Cornelsen.

Ehmke, T., Duchhardt, C., Geiser, H., Grüßing, M., Heinze, A., \& Marschick, F. (2009). Kompetenzerhebung über die Lebensspanne - Erhebung von mathematischer Kompetenz im Nationalen Bildungspanel. In A. Heinze \& M. Grüßing (Hrsg.), Mathematiklernen vom Kindergarten bis zum Studium: Kontinuität und Kohärenz als Herausforderung für den Mathematikunterricht (S. 313-327). Münster: Waxmann.

Gniewosz, B. (2015). Kompetenzentwicklung. In H. Reinders, H. Ditton, C. Gräsel, \& B. Gniewosz (Hrsg.), Empirische Bildungsforschung. Gegenstandsbereiche (2. Aufl., S. 69-81). Wiesbaden: Springer VS.

Goldhammer, F., \& Hartig, J. (2007). Interpretation von Testresultaten und Testeichung. In H. Moosbrugger \& A. Kelava (Hrsg.), Testtheorie und Fragebogenkonstruktion (S. 165-192). Heidelberg: Springer.

Hartig, J., \& Klieme, E. (2006). Kompetenz und Kompetenzdiagnostik. In K. Schweizer (Hrsg.), Leistung und Leistungsdiagnostik (S. 127-144). Heidelberg: Springer.

IBM Corp (2013). SPSS statistics for windows (version 22). Armonk: IBM Corp.

IQB (2012) = Institut zur Qualitätsentwicklung im Bildungswesen. (2012). Kompetenzstufenmodell zu den Bildungsstandards für den Hauptschulabschluss und den Mittleren Schulabschluss im Fach Mathematik. http://www.iqb.hu-berlin.de/bista/bista/ksm/Kompetenzstufenm_1.pdf. Zugegriffen: 11. Aug. 2017.

Kampa, N., Köller, O., Schmidt, F. T.C., \& Leucht, M. (2016a). Kompetenzen im Fach Mathematik. In M. Leucht, N. Kampa \& O. Köller (Hrsg.), Fachleistungen beim Abitur: Vergleich allgemeinbildender und beruflicher Gymnasien in Schleswig-Holstein (S. 119-143). Münster: Waxmann.

Kampa, N., Leucht, M., \& Köller, O. (2016b). Mathematische Kompetenzen in unterschiedlichen Profilen der gymnasialen Oberstufe. In J. Kramer, M. Neumann, \& U. Trautwein (Hrsg.), Abitur und Matura im Wandel. Historische Entwicklungslinien, aktuelle Reformen und ihre Effekte (Edition ZfE Bd. 2, S. 161-188). Wiesbaden: Springer VS.

Kampa, N., Wagner, H., \& Köller, O. (in Vorbereitung). Validity Arguments for Standard-Setting Procedures - The Case of Two Angoff Method Variations.

Klieme, E., Maag-Merki, K., \& Hartig, J. (2007). Kompetenzbegriff und Bedeutung von Kompetenzen im Bildungswesen. In J. Hartig \& E. Klieme (Hrsg.), Möglichkeiten und Voraussetzungen technologie- 
basierter Kompetenzdiagnostik. Eine Expertise im Auftrag des Bundesministeriums für Bildung und Forschung (Bd. 20, S. 5-16). Bonn: Bundesministerium für Bildung und Forschung (BMBF).

KMK (2003) = Kultusministerkonferenz. (2003). Bildungsstandards im Fach Mathematik für den Mittleren Schulabschluss. München: Luchterhand.

KMK (2012) = Kultusministerkonferenz (2012). Bildungsstandards im Fach Mathematik für die Allgemeine Hochschulreife. München: Luchterhand.

Kolen, M. J., \& Brennan, R. L. (Hrsg.) (2004). Test equating, scaling, and linking: methods and practices. New York: Springer.

Köller, O. (2016a). Alternative Wege zur Hochschulreife. In M. Leucht, N. Kampa \& O. Köller (Hrsg.), Fachleistungen beim Abitur: Vergleich allgemeinbildender und beruflicher Gymnasien in SchleswigHolstein (S. 33-53). Münster: Waxmann.

Köller, O. (2016b). Soziale Herkunft und kognitive Grundfähigkeiten der Schülerinnen und Schüler. In M. Leucht, N. Kampa, \& O. Köller (Hrsg.), Fachleistungen beim Abitur: Vergleich allgemeinbildender und beruflicher Gymnasien in Schleswig-Holstein (S. 99-114). Münster: Waxmann.

Köller, O., \& Baumert, J. (2012). Schulische Leistungen und ihre Messung. In W. Schneider \& U. Lindenberger (Hrsg.), Entwicklungspsychologie (S. 645-661). Weinheim: Beltz.

Köller, O., Baumert, J., \& Schnabel, K. (1999). Wege zur Hochshchulreife: Offenheit des Systems und Sicherung vergleichbarer Standards. Analysen am Beispiel der Mathematikleistungen von Oberstufenschülern an integrierten Gesamtschulen und Gymnasien in Nordrhein-Westfalen. Zeitschrift für Erziehungswissenschaft, 2, 370-405.

Köller, O., Watermann, R., Trautwein, U., \& Lüdtke, O. (2004). Wege zur Hochschulreife in Baden Württemberg: TOSCA - Eine Untersuchung an allgemein bildenden und beruflichen Gymnasien. Opladen: Leske + Budrich.

Köller, O., Baumert, J., Cortina, K., \& Trautwein, U. (2010). Bildungsverläufe und psychosoziale Entwicklung im Jugendalter und im jungen Erwachsenenalter. In C. Spiel, B. Schober, P. Wagner, \& R. Reimann (Hrsg.), Bildungspsychologie (S. 245-252). Göttingen: Hogrefe.

Lee, J. (2010). Tripartite growth trajectories of reading and math achievement: tracking national academic progress at primary, middle, and high school levels. American Educational Research Journal, 47, 800-832. https://doi.org/10.3102/0002831210365009.

Leucht, M., \& Köller, O. (2016). Anlage und Durchführung der Studie. In M. Leucht, N. Kampa, \& O. Köller (Hrsg.), Fachleistungen beim Abitur: Vergleich allgemeinbildender und beruflicher Gymnasien in Schleswig-Holstein (S. 79-93). Münster: Waxmann.

Leucht, M., Kampa, N., \& Köller, O. (2016). Fachleistungen beim Abitur - Vergleich allgemeinbildender und beruflicher Gymnasien in Schleswig-Holstein. Münster: Waxmann.

Muthén, L. K., \& Muthén, B.O. (1998). Mplus user's guide (Sixth). Los Angeles: Muthén \& Muthén.

Nissen, A., Ehmke, T., Köller, O., \& Duchhardt, C. (2015). Comparing apples with oranges? An approach to link TIMSS and the National Educational Panel Study in Germany via equipercentile and IRT methods. Studies in Educational Evaluation, 47, 58-67.

Pant, H. A., Stanat, P., Schroeders, U., Roppelt, A., Siegle, T., \& Pöhlmann, C. (2013). IQB-Ländervergleich 2012. Münster: Waxmann.

Retelsdorf, J., Nagy, G., \& Köller, O. (2017). Lernausgangslagen Auszubildender in Berufen mit hohen mathematisch-naturwissenschaftlichen Anforderungen. Unterrichtswissenschaft, 45, 5-21.

Roppelt, A., Blum, W., \& Pöhlmann, C. (2013). Die im Ländervergleich 2012 untersuchten mathematischen und naturwissenschaftlichen Kompetenzen. In H. A. Pant, P. Stanat, U. Schroeders, A. Roppelt, T. Siegle, \& C. Pöhlmann (Hrsg.), IQB-Ländervergleich 2012. Mathematische und naturwissenschaftliche Kompetenzen am Ende der Sekundarstufe I (S. 23-52). Münster: Waxmann.

Trautwein, U., Köller, O., Lehmann, R., \& Lüdtke, O. (2007). Schulleistungen von Abiturienten. Regionale, schulformbezogene und soziale Disparitäten. Münster: Waxmann.

Trautwein, U., Neumann, M., Nagy, G., Lüdtke, O., \& Maaz, K. (2010). Schulleistungen von Abiturienten. Die neu geordnete gymnasiale Oberstufe auf dem Prüfstand. Wiesbaden: Springer VS.

Watermann, R., Nagy, G., \& Köller, O. (2004). Mathematikleistungen in allgemein bildenden und beruflichen Gymnasien. In O. Köller, R. Watermann, U. Trautwein, \& O. Lüdtke (Hrsg.), Wege zur Hochschulreife in Baden-Württemberg: TOSCA - Eine Untersuchung an allgemeinbildenden und beruflichen Gymnasien (S. 205-283). Opladen: Leske + Budrich.

Wu, M., Adams, R. J., Wilson, M.R., \& Haldane, S. A. (2007). ACER ConQuest version 2: Generalised item response modelling software. Camberwell: Australian Council for Educational Research. 